\title{
Effect of Lattice Strain on Measured Thermal Properties of Fe Nanoparticles - An X-ray Diffraction Study
}

\author{
Endla Purushotham \\ Department of Physics, S R Engineering College (Autonomous), Warangal, India
}

Email address:

psm45456@gmail.com

To cite this article:

Endla Purushotham. Effect of Lattice Strain on Measured Thermal Properties of Fe Nanoparticles - An X-ray Diffraction Study. International Journal of Fluid Mechanics \& Thermal Sciences. Vol. 5, No. 3, 2019, pp. 63-66. doi: 10.11648/j.ijfmts.20190503.11

Received: April 7, 2019; Accepted: July 19, 2019; Published: August 6, 2019

\begin{abstract}
In this paper, nano Fe particles have been produced by ball milling process. Fe powder was ball milled in an argon inert atmosphere Ball milling was carried out for the total duration of 20 hours. The sample was taken out after every 4 hours of milling and it was characterized for its particle size, lattice strain, and Debye-Waller factor, root mean square amplitudes of vibration by X-ray powder diffraction. The high-energy ball milling of Fe after 20 hours resulted in particle size of $39.44 \mathrm{~nm}$ and lattice stain 0.68 Lattice strains in Fe powder produced by milling have been analyzed by X-ray powder diffraction. The lattice strain $(\varepsilon)$ and Debye-Waller factor (B) are determined from the half-widths and integrated intensities of the Bragg reflections. Debye-Waller factor is found to increase with the lattice strain. From the correlation between the strain and effective Debye-Waller factors have been estimated for Fe. The variation of energy of vacancy formation as a function of lattice strain has been studied. The lattice strain $(\varepsilon)$ and Debye-Waller factor $(B)$ are determined from the half-widths and integrated intensities of the Bragg reflections. In Fe, the Debye-Waller factor is found to increase with the lattice strain. From the correlation between the strain and effective Debye-Waller factor, the Debye-Waller factors for zero strain have been estimated for $\mathrm{Fe}$. The variation of energy of vacancy formation as a function of lattice strain has been studied. As grinding time increases energy of vacancy formation decreases. However, the milling produces lattice strain and also enhances the effective Debye-Waller factor. By an extrapolation of the plot between the Debye-Waller factor and the lattice strain, the zero strain Debye-Waller factors are obtained for Fe. The variation of energy of vacancy formation as a function of lattice strain has been studied.
\end{abstract}

Keywords: Ball Milling, X-ray Diffraction, Particle Size, Lattice Strain, Debye-Waller Factor, Vacancy Formation Energy

\section{Introduction}

The Debye temperature and Debye-Waller factor is an important lattice dynamical property. There is considerable $\mathrm{X}$-ray work on the Debye-Waller factors of Fe [1-4]. But it is interesting to study the effect of lattice strains on the DebyeWaller factors of these metals. Inagaki et al $[5,6]$ showed that in several non-metallic powders, the strains produced during grinding have a significant effect on the Debye-Waller factors measured from X-ray diffraction intensities. Sirdeshmukh et al [7] observed the effect of lattice strains on the Debye-Waller factors in semiconductor powder materials. Gopi Krishna and Sirdeshmukh [8] studied the effect of lattice strains on the Debye-Waller factor of ytterbium metal. In the present investigation the results of a systematic study of the effect of lattice strains on the Debye-Waller factors and particle size of face centred cubic Fe metal is reported.

\section{Experimental}

Powder samples were obtained by gently filing highly pure $\mathrm{Fe}$ metal ingots with a jeweller's file. A part of this powder was used to prepare the initial sample. The remaining powder was subjected to milling in a ball mill for 5, 10, 15 and 20 hours to produce strains and decrease the particle size. X-ray diffractograms were recorded with the initial sample and with samples prepared after each spell of milling. The diffractograms were obtained with a Philips CWU 3710 Xray powder diffractometer in the $2 \theta$ range $20-120^{\circ}$ using filtered $\mathrm{CuK}_{\alpha}$ at a goniometer speed of $0.5^{\circ}$ per minute and a chart speed of $20 \mathrm{~mm} / \mathrm{min}$. All measurements were made at room temperature. The observed integrated intensities have 
been corrected for thermal diffuse scattering using the method of Chipman and Paskin [9].

\section{Analysis of Data}

Lattice strain and Particle size determination

When the size of the individual crystals is less than about $100 \mathrm{~nm}$ the term "particle size" is usually used. When the crystallites of a material are smaller than $100 \mathrm{~nm}$, they have too small a number of parallel diffraction planes and so they produce broadened diffraction peaks instead of a sharp peak. Lattice strain present in the sample is another cause of broadening of Bragg diffraction peaks. In addition to this, there are instrumental factors such as unresolved $\alpha_{1}$ and $\alpha_{2}$ peaks, imperfect focusing which lead to the line broadening. There are various methods in practice to estimate the particle size. X-ray diffraction is a simpler and easier approach for the determination of precise particle size and the lattice strain in powder samples. The principle involved in the X-ray diffraction approach is precise quantification of the broadening of the Bragg diffraction peaks. Scherrer equation, Hall-Williamson method and Warren-Averbach method are some of the techniques based on this principle. Of the above methods, Scherrer equation method for the estimation of particle size does not take into account the broadening due to lattice strain present in the sample. As such in the present investigation, the lattice strains have been estimated using Hall-Williamson method. Recently, Bharati et al [10] have used this method to estimate the lattice strain and particle sizes of silver nano particles and composite silver nano particles. In this method the integral breadth of the diffraction peak is determined. The integral breadth is given by the integrated intensity divided by the maximum intensity. Thus, the observed peak broadening $\mathrm{B}_{\mathrm{o}}$ may be represented as

$$
\mathrm{B}_{\mathrm{o}}=\mathrm{B}_{\mathrm{i}}+\mathrm{B}_{\mathrm{r}}
$$

where $B_{o}$ is the observed peak broadening in radians, $B_{i}$ is the instrumental broadening in radians and $B_{r}$ is the broadening due to the small particle size and lattice strain. The instrumental broadening has been estimated using a pure strain-free fine sodium chloride powder sample subjected to $\mathrm{XRD}$ under identical conditions as those for the strained metallic powders. Eq. (1) holds good if the diffraction peaks exhibit purely Cauchy profile. However, when the diffraction peaks are partly Cauchy and partly Gaussian for profiles, the following relation between $\mathrm{B}_{0}, \mathrm{~B}_{\mathrm{i}}$ and $\mathrm{B}_{\mathrm{r}}$ holds good, Bharati et al [10].

$$
\mathrm{B}_{\mathrm{r}}=\left[\left(\mathrm{B}_{\mathrm{o}}-\mathrm{B}_{\mathrm{i}}\right)\left(\mathrm{B}_{\mathrm{o}}{ }^{2}-\mathrm{B}_{\mathrm{i}}{ }^{2}\right)^{1 / 2}\right]^{1 / 2}
$$

Now, according to Scherrer equation, the broadening due to small particle size may be expressed as

$$
\mathrm{Bc}=\frac{\mathrm{k} \lambda}{\mathrm{t}} \cos \theta
$$

where $B_{c}$ is the broadening solely due to small crystallite size, $\mathrm{K}$ a constant whose value depends on particle shape and usually taken as unity, $\mathrm{t}$ the crystallite size in nanometers, $\theta$ the Bragg angle and $\lambda$ is the wavelength of incident X-ray beam in nanometers. Similarly, according to Wilson [11], the broadening due to lattice strain may be expressed by the relation,

$$
\mathrm{B}_{\mathrm{s}}=\varepsilon \tan \theta
$$

where $\mathrm{B}_{\mathrm{s}}$ is the peak broadening due to lattice strain and $\varepsilon$ the strain distribution within the material and $\theta$ is the Bragg angle. Based on Eqs. (3) and (4) the total peak broadening $B_{r}$ may be expressed as,

$$
\mathrm{B}_{\mathrm{r}}=\frac{\mathrm{k} \lambda}{\mathrm{t} \cos \theta}+\varepsilon \tan \theta
$$

which can be written

$$
\text { as } \mathrm{B}_{\mathrm{r}} \cos \theta=\frac{\mathrm{k} \lambda}{\mathrm{t}}+\varepsilon \sin \theta
$$

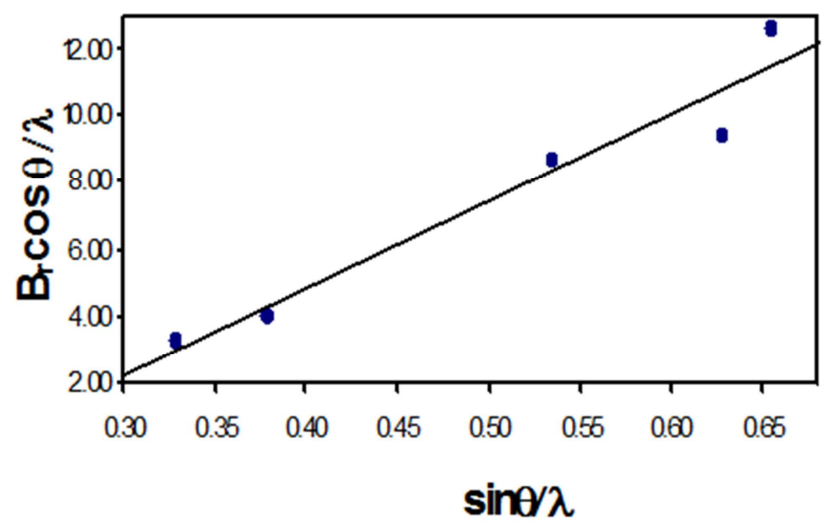

Figure 1. Plot of $B_{r} \cos \theta / \lambda V_{s} \sin \theta / \lambda$ for Fe after milling for 10 hours.

Figure 1: The plot of $B_{r} \cos \theta / \lambda$ versus $\sin \theta / \lambda$ is a straight line with slope equal to $\varepsilon$ and hence the particle size ' $t$ ' can be estimated from the intercept. Typical Hall-Williamson plot between $B_{r} \cos \theta / \lambda$ and $\sin \theta / \lambda$ is shown in Figure 1 . By a least square treatment of data, $\mathrm{B}$ was determined.

Debye Waller factor, Debye temperature and mean square amplitude of vibration determination:

For the relative intensity method, the expression for the observed intensities $\mathrm{I}_{0}$ is given by

$$
\mathrm{I}_{0}=\mathrm{CL}_{\mathrm{p}} \mathrm{JF}_{\mathrm{T}}^{2}
$$

Where $\mathrm{L}_{\mathrm{p}}$ is the Lorentz-polarization factor, $\mathrm{J}$, the multiplicity factor, $\mathrm{F}_{\mathrm{T}}$ the structure factor and $\mathrm{C}$ is a constant. For a flat powder specimen, the absorption correction is independent of the angle $\theta$, Klug and Alexander [12] and, hence, is lumped with the constant. The structure factor $F_{T}$ in terms of the structure factor $F$ for the static lattice is given by

$$
\mathrm{F}_{\mathrm{T}}=\mathrm{Fe}^{-\mathrm{M}}
$$

We may also write Eq. (7) as 


$$
\mathrm{I} 0=I_{c} e^{-2 B\left(\frac{\sin \theta}{\lambda}\right)^{2}}
$$

Where $I_{c}$ is the intensity corresponding to the static lattice and is given by

$$
\mathrm{I}_{\mathrm{c}}=\mathrm{L}_{\mathrm{p}} \mathrm{JF}^{2}
$$

For crystals with fcc structure, the structure factor $F$ is given by

$$
\mathrm{F}=4 \mathrm{f}
$$

$\mathrm{f}$ being the atomic scattering factor. Values of the atomic scattering factor were taken from Cromer and Waber [13] and International Tables for X-ray Crystallography [14], and have been corrected for dispersion, Cromer and Liberman [15]. The choice of the atomic scattering factors was guided by Cromer's [13] recommendation who suggested that the atomic scattering factors given in the International Tables for X-ray Crystallography [14] which are computed from the Hartree-Fock wave functions are the best for the lighter atoms or ions in the periodic table up to $\mathrm{Rb}^{+1}$. For the heavier atoms or ions Cromer recommends the use of the Dirac-slater relativistic scattering factors given by Cromer and Waber [13].

From Eq. (9) it can be seen that $\log \left(\mathrm{I}_{0} / \mathrm{I}_{\mathrm{c}}\right)$ is linearly related to $(\sin \theta / \lambda)^{2}$. By a least square treatment of data, $B$ was determined. From the Debye-Waller theory

$$
\mathrm{B}=\left(\frac{8 \pi^{2}}{3}\right)<\mathrm{u}^{2}>
$$

for a cubic crystal, where $<\mathrm{u}^{2}>$ is the mean-square amplitude of vibration. Further, B, may also be expressed as

$$
\mathrm{B}=\left(\frac{6 h^{2}}{m k_{B} T}\right) \mathrm{W}(\mathrm{x})
$$

where $\mathrm{m}$ is the mass, $\mathrm{T}$ the absolute temperature and $\mathrm{h}$ and $\mathrm{k}_{\mathrm{B}}$ are the Planck and the Boltzmann constants respectively. The function $\mathrm{W}(\mathrm{x})$ is given by

$$
\mathrm{W}(\mathrm{x})=\left[\frac{\varphi(x)}{x^{2}}+\frac{1}{4 x}\right]
$$

where $\varnothing(x)$ is the Debye function and $x=\theta_{M} / T, \theta_{M}$ being the Debye temperature. Benson and Gill [16] have tabulated values of $\mathrm{W}(\mathrm{x})$ for a wide range of $\mathrm{x}$ for small increments, from which $\theta_{M}$ can be obtained from the value of $B$.

\section{Results and Discussion}

Table 1: The values of the lattice strain $(\varepsilon)$, particle size $(t)$, root mean square amplitude of vibrations $<\mathrm{u}>$, Debye-Waller factor (B) and Debye temperature $\left(\theta_{M}\right)$ of Fe powders, ground for different durations, obtained in the present study are given in Table 1. Particle size decrease with milling time. Both lattice strain and Debye-Waller factor increase with milling time. While comparing the Debye-Waller factors calculated from the lattice dynamical models with experimental results, Vetelino et al [17] have attributed the difference to inaccuracies in the experimental values caused by neglecting the TDS corrections.

The energy of vacancy formation $\left(E_{f}\right)$ and the Debye temperature $(\theta)$ of a solid is given by

$$
\mathrm{E}_{\mathrm{f}}=\mathrm{A}(\mathrm{k} / \mathrm{h})^{2} \mathrm{M} \theta^{2} \mathrm{a}^{2}
$$

From this relation, the variation of vacancy formation energy as a function of lattice strain in Fe has been calculated and also included in Table 1.

\begin{tabular}{|c|c|c|c|c|c|c|c|}
\hline Metal & Grinding time & $\varepsilon \times 10^{3}$ & $t(n m)$ & $<\mathbf{u}>(\AA)$ & $\mathbf{B}\left(\AA^{2}\right)$ & $\theta_{M}(K)$ & $E_{f}(e V)$ \\
\hline \multirow[t]{5}{*}{$\mathrm{Fe}$} & 0 & 0.19 & 162.46 & 0.0025 & 0.22 & 289 & 6.26 \\
\hline & 5 & 0.23 & 135.30 & 0.0035 & 0.28 & 281 & 5.86 \\
\hline & 10 & 0.36 & 69.72 & 0.0082 & 0.64 & 184 & 2.88 \\
\hline & 15 & 0.49 & 58.49 & 0.0146 & 1.15 & 138 & 1.83 \\
\hline & 20 & 0.68 & 39.44 & 0.0239 & 1.88 & 108 & 0.97 \\
\hline
\end{tabular}

As grinding time increases particle size decreases and lattice strain increases. For o hours calculated lattices strain $0.19 \times 10^{3}$ and particle size $162.46 \mathrm{~nm}$, for 20 hours obtained lattices strain $0.68 \times 10^{3}$ and particle size $39.44 \mathrm{~nm}$.

Table 1. Values of lattice strain $(\varepsilon)$, particle size $(t)$, Debye-Waller factor $(B)$, root mean square amplitudes of vibration $\langle u\rangle$, Debye temperature $\left(\theta_{M}\right)$ and energy of vacancy formation $\left(E_{f}\right)$ of strained Fe powders.

\section{Conclusion}

Fe powders were strained by milling for 20 hours. From a study of X-ray diffractograms recorded at different stages of milling, it is observed that milling for 20 hours has systematic effect on the particle size. However, the milling produces lattice strain and also enhances the effective DebyeWaller factor. By an extrapolation of the plot between the Debye-Waller factor and the lattice strain, the zero strain Debye-Waller factors are obtained for Fe. The variation of energy of vacancy formation as a function of lattice strain has been studied.

\section{References}

[1] Batterman, B. W., Chiman, D. R. and De March, J. J. (1961). Phys. Rev. 122, 68.

[2] Paakkari, T. (1974). Acta Cryst. A30, 83.

[3] Radchenko, M. E. and Tsvetkov, V. P. (1965). Ukr. fiz. Zh. 10, 99. 
[4] Mohanlal, S. K. (1979). J. Phys. C. 12, 2651.

[5] Inagaki, M. Furuhashi, H., T Ozeki et al., J Mater Sci. 6, (1971) 1520.

[6] M Inagaki, H Furuhashi, T Ozeki and S Naka J. Mater. Sci. 8, (1973) 312 .

[7] Sirdeshmukh, D. B., Subhadra, K. G., Hussain, K. A., Gopi Krishna, N., and Rag-havendra Rao. B., Cryst. Res. Technol 28, (1993) 15.

[8] Gopi Krishna, N., and Sirdeshmukh., D. B., Indian J Pure \& Appl Phys. 31, (1993) 198.

[9] Chipman, D. R., and Paskin, A., J. Appl. Phys. 30, (1959) 1938.

[10] Gopi Krishna, N and Sirdeshmukh, D. B. Indian J Pure \& Appl Phys. 31, 198 (1993).
[11] Gopi Krishna, N et al, Indian J Phys. 84 (7), 887 (2010).

[12] Klug, H. P and Alexande L E, X-ray Diffraction Procedures (John Wiley and Sons, U.S.A.) (1974).

[13] Cromer D T and Waber J T, Acta Cryst. 18104 (1965).

[14] International Tables for X-ray Crystallography Vol. III (Kynoch Press, Birmingham) (1968).

[15] Cromer D T and Liberman D, J. Chem. Phys. 531891 (1970).

[16] Benson G. C, and Gill E. K, Table of Integral Functions Related to Debye-Waller factor, National Research Council of Canada, Ottawa. (1966).

[17] Vetelino, J. F., Gaur, S. P., Mitra, S. S., Phys. Rev. B5, (1972) 2360. 